\title{
Efficient height reduction over-the-cell channel router
}

\author{
P.-W. Shew \\ P.-Y. Hsiao \\ Y.-C. Lim
}

Indexing terms: Algorithms, Network routing, Over-the-cell channel routers, VLSI

Abstract: The authors present a new algorithm for both two-layer and three-layer over-the-cell channel routing in the standard cell VLSI design. The approach exploits vacant terminals on the channel boundary effectively. It considers the following factors simultaneously to select net segments for routing over the cells: density distribution in the channel, the longest path in the vertical constraint graph, elimination of cycles in the vertical constraint graph and reduction in maximum cliques in the horizontal constraint graph. With respect to the PRIMARY 1 benchmark examples, the router achieved a $41.3 \%$ improvement over the Greedy channel router (one without using over-the-cell area) for a two-layer routing model and a $61.0 \%$ improvement for a three-layer routing model. This outperforms all previous algorithms.

\section{Introduction}

In VLSI layout design using standard cells, channel routing is a crucial problem which has been studied extensively. A good routing solution should complete the connections in the channel with the minimum number of tracks. This will minimise the channel height, thus reducing the total chip area. Fig. $1 a$ shows an example of routing between two rows of standard cells (i.e. in the channel area) within a circuit.

Many channel routing algorithms have been proposed and they can produce solutions very close to optimum (e.g. the Greedy channel router [1]). Researchers have also proposed the use of the area over the standard cells for routing in second (M2) and third (M3) metal layers (the first metal (M1) is usually used for routing within the cell). Such routing style is known as over-the-cell channel routing. Fig. $1 b$ illustrates such a routing style. Efficient utilisation of the area over the cells can reduce the layout area substantially. The reduction in chip area with this approach is even more significant in today's fabrication technology, as more metal layers are available for routing.

It has been shown that the channel routing problem is NP-hard [2]. Over-the-cell (OTC) channel routing is a more general case of the channel routing problem, hence it is obviously NP-hard too. A number of heuristics have been proposed [3-12] for either a two-layer (i.e. using only M2) or a three-layer (i.e. using both M2 and M3) OTC channel routing model. After the introduction of using vacant terminals in OTC routing [8], recent OTC channel routers can reduce the channel height by $30 \%$ for a two-layer model and $50 \%$ for a three-layer model (see Table 1).

In this paper we present a new algorithm which we have implemented for two-layer and three-layer OTC
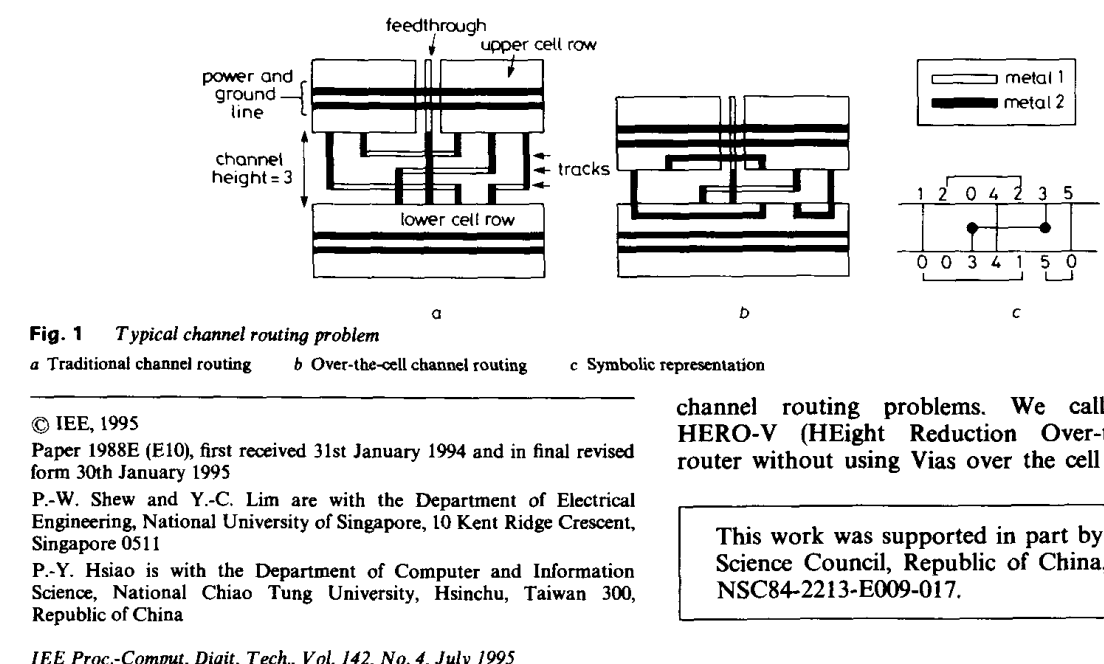

1 Typical channel routing problem

\section{(C) IEE, 199}

(1) January 1994 and in final revised Singapore 0511 channel routing problems. We call our program HERO-V (HEight Reduction Over-the-cell channel router without using Vias over the cell area). The algo-

This work was supported in part by the Nationa Science Council, Republic of China, under grant NSC84-2213-E009-017. 
Table 1: Comparison with other routers for PRIMARY 1

\begin{tabular}{|c|c|c|c|c|c|c|c|c|c|c|}
\hline \multirow[t]{3}{*}{ Channel } & \multirow[t]{3}{*}{$\%$ Vacancy } & \multicolumn{9}{|c|}{ Final channel height } \\
\hline & & \multicolumn{5}{|c|}{ Two-layer } & \multicolumn{4}{|c|}{ Three-layer } \\
\hline & & $\mathrm{Gr}[1]$ & OTC-I [10] & WISER [8] & WILMA [12] & HERO.V & Gr [1] & WILMA [12] & $O-V[11]$ & HERO-V \\
\hline 1 & 85 & 11 & 6 & 5 & 2* & 4 & 6 & 2 & $1^{*}$ & $1^{*}$ \\
\hline 2 & 75 & 16 & 14 & 12 & 11 & $10^{*}$ & 9 & 5 & 5 & $4^{*}$ \\
\hline 3 & 64 & 21 & 16 & 16 & 16 & $14^{*}$ & 11 & 6 & 6 & $5^{*}$ \\
\hline 4 & 60 & 24 & 20 & 21 & 20 & $18^{*}$ & 15 & 9 & $6^{*}$ & 7 \\
\hline 5 & 64 & 21 & 17 & 17 & 17 & $13^{*}$ & 11 & 7 & 6 & $5^{*}$ \\
\hline 6 & 53 & 23 & 17 & 18 & 17 & $16^{*}$ & 12 & 7 & 7 & $6^{*}$ \\
\hline 7 & 58 & 22 & 14 & 14 & 13 & $12^{*}$ & 14 & 5 & $4^{*}$ & 4* \\
\hline 8 & 51 & 24 & 17 & 18 & 17 & $15^{*}$ & 16 & 7 & 7 & 6* \\
\hline 9 & 54 & 21 & 15 & 16 & 16 & $12^{*}$ & 13 & 6 & 6 & $5^{*}$ \\
\hline 10 & 61 & 15 & 10 & 11 & 10 & $9^{*}$ & 8 & 5 & $4^{*}$ & 4* \\
\hline 11 & 64 & 17 & 11 & 12 & 11 & $10^{*}$ & 12 & 5 & $3^{*}$ & $3^{*}$ \\
\hline 12 & 64 & 15 & 10 & 11 & 10 & $9^{*}$ & 8 & 4 & $3^{*}$ & 4 \\
\hline 13 & 63 & 13 & 10 & 9* & $9^{*}$ & $9^{*}$ & 8 & $3^{*}$ & $3^{*}$ & $3^{*}$ \\
\hline 14 & 64 & 13 & 11 & 9 & 9 & $7 *$ & 7 & 4 & $3^{*}$ & $3^{*}$ \\
\hline 15 & 63 & 11 & 7 & 7 & 6 & $5^{*}$ & 6 & 2* & $2^{*}$ & $2 *$ \\
\hline 16 & 68 & 11 & 8 & 7 & 6 & 4* & 6 & $2^{*}$ & $2^{*}$ & 2* \\
\hline 17 & 68 & 14 & 9 & 8 & 8 & $7^{*}$ & 7 & $3^{*}$ & $3^{*}$ & $3^{*}$ \\
\hline 18 & 92 & 6 & 4 & 3 & 3 & $1 *$ & 3 & 1 & $0^{*}$ & $0^{*}$ \\
\hline$\Sigma$ & 65 & 298 & 216 & $214 \dagger$ & 201 & $175^{*}$ & 172 & 83 & 71 & $67^{*}$ \\
\hline$\Delta \%$ & - & - & 28.9 & 29.6 & 32.6 & $41.3^{*}$ & - & 51.7 & 58.7 & $61.0^{*}$ \\
\hline
\end{tabular}

Best result for each channel

† Addition error in References 8 and 9

rithm has a generic nature in that it may be further extended to multiple-layer OTC channel routing. Our approach efficiently selects a subset of net segments to be routed over the cells with consideration of density distribution in the channel, and by making use of the vacant terminals it reduces the longest path in the vertical constraint graph, as well as the maximum cliques in the horizontal constraint graph. At the same time, cycles in the vertical constraint graph are eliminated as much as possible. We have tested our algorithm with a number of benchmark examples to verify its effectiveness. For the PRIMARY 1 example, our algorithm achieved $41.3 \%$ and $61.0 \%$ reduction in total channel height for two-layer and three-layer OTC routing models, respectively. It outperforms all previous algorithms.

\section{Physical model for OTC routing}

The OTC channel routing is restricted by the number of metal layers available in the IC fabrication. In this paper, we assume that the intracell connections are done in the polysilicon and the first metal (M1) layer. The feedthrough is made in the M1 layer too. The power and ground lines are laid in the second metal (M2) layer along the centre of the area over the standard cells. These lines divide the over-the-cell (OTC) area into two halves and the cell height permits us to route six tracks in the M2 layer over each half. The OTC area in the third metal (M3) layer is not divided, but for simplicity we split the area into two halves and only route, at most, seven tracks over each half. Vias may or may not be made in the OTC area, depending on the fabrication technology. In our current implementation, we assume vias are not allowed so OTC routing in $\mathrm{M} 2$ and $\mathrm{M} 3$ layers must be planar.

As for routing in the channel region, we adopt the traditional HV and HVH models for two-layer and threelayer channel routing. Under these channel routing models, horizontal and vertical wire segments are used exclusively in alternating metal layers. We make use of doglegs to complete the channel routing while keeping the final channel height as low as possible. Fig. $1 b$ illustrates this physical routing model. Discussion on other
OTC routing models can be found in Reference 5. Fig. 1c shows a symbolic representation of the same problem as in Figs. $1 a$ and $b$.

\section{Terminology and notation}

Amongst the $N_{\text {net }}$ nets in a given channel, some may be multiterminal, i.e. a net consisting of more than two terminals. The approach in this paper first decomposes all multiterminal nets into modified two-terminal net segments adjacent at common terminal locations. Each pair of terminals defines a net segment which will either be routed in the channel or in the OTC area. Thus net $n_{i}=\left\{t_{t, 1}, t_{b, 4}, t_{t, 7}\right\}$ (Fig. 2), after being decomposed, gives us the segments $s_{i}=\left\{s_{i, 1}, s_{i, 2}\right\}$, where $t_{\mathrm{s}, c}$ refers to the terminal on row $s$ (TOP or BOTTOM) at column $c$; $s_{i, j}=\left(l_{i, j}, r_{i, j}\right)$ where $l_{i, j}$ and $r_{i, j}$ denote the left and right column of the net segment $s_{i, j}$.

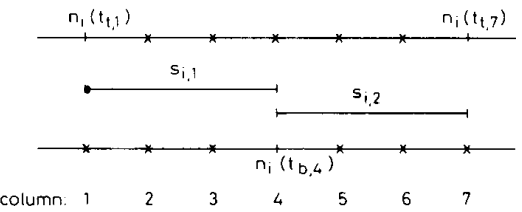

Fig. 2 Decomposing multiterminal net, $n_{i}$, into 2 two-terminal nets Fig. 2 Decomposing multitem
with net segments $s_{i, 1}$ and $s_{i, 2}$

Next we define the set of critical columns, $c_{r}=\left\{c_{r_{i}}\right.$; $\left.(1 \leqslant i \leqslant N)\left[D\left(c_{r i}\right)=D_{0}\right]\right\}$, where $N$ is the number of columns in the channel, $D(c)$ is the local density at column $c$ (i.e. the number of net segments intersecting column $c$ ), and the channel density, $D_{0}=\max [D(c)]$ Our approach considers the horizontal constraints in the channel by analysing the modified horizontal constraint graph (MHCG). MHCG is an undirected graph $G_{m h}=$ $\left(V^{\prime}, E_{m h}\right)$ where $V^{\prime}=\left\{v_{i, j}: v_{i, j}\right.$ represents the modified net segment $\left.s_{i, j}\right\}$ and $E_{m h}=\left\{\left(v_{i, j}, v_{k, l}\right): s_{i, j}\right.$ intersects $s_{k, l}$ segment $\left.s_{i, j}\right\}$ and $E_{m h}=\left\{\left(v_{i, j}, v_{k, j}\right): s_{i, j}\right.$ intersects $s_{k, l}$,
$i \neq k\}$. The vertical constraints, however, are considered by analysing the longest path in the vertical constrain

IEE Proc.-Comput. Digit. Tech., Vol. 142, No. 4, July 1995 
graph (VCG). VCG is a directed graph, $G_{v}=\left(V, E_{v}\right)$ where $V=\left\{v_{i}: v_{i}\right.$ represents net $\left.i\right\}$ and $E_{p}=\left\{\left(v_{i}, v_{j}\right)\right.$ : $(\exists 1 \leqslant c \leqslant N)[\operatorname{TopNet}(c)=i, \operatorname{BottomNet}(c)=j, i \neq j]\}$. TopNet $(c)$ and BottomNet $(c)$ are the top terminal net and bottom terminal net at column $c$, respectively.

\section{Reduction in channel height}

As mentioned earlier, OTC channel routing can reduce the channel height of a circuit. Fig. 3 depicts nine valid OTC routing types for a net segment $s_{i, j}$. These types are not exhaustive but are the ones considered in our algorithm. Note that we allow combinations of these types for any net segment remaining in the channel. In Section 4.1, we discuss the factors which affect the channel height of a channel routing solution (without OTC routing). Then we describe the heuristic cost function befitting our consideration of all these factors in Section 4.2 and we give a summary of our algorithm in Section 5. To aid the description of our algorithm, we trace the execution of our program for a simple problem in Fig. 4.

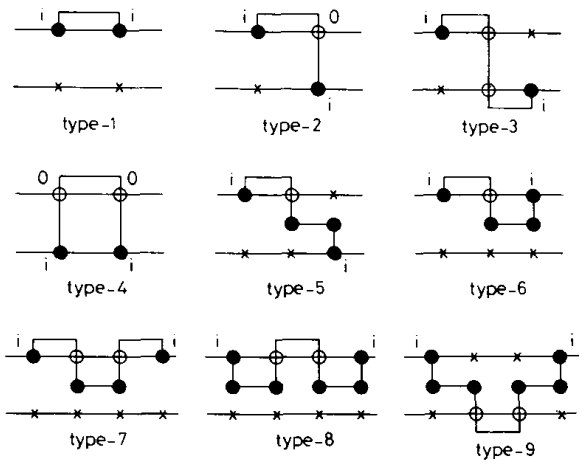

Fig. 3 Nine valid types of OTC routing

4.1 Lower bound for channel height

The first lower bound for channel height is the channel density $d$. In earlier algorithms (e.g. in References 4-7 and 10 ), the reduction in channel density was the ultimate goal. However, without considering other factors, they did not perform well. The second lower bound is the length of the longest path in VCG $l$. It is possible to reduce $l$ by doglegging [13] or making OTC routing as illustrated in Fig. $4 c$. The weight $w_{2}$ in our heuristic cos function is improvised to achieve the lowest value of $l$. In addition to $\max (d, l)$, Brown and Rivest [14] have shown that for a two-terminal net routing problem $\left.t \geqslant-(w-n)+\Gamma \sqrt{ }\left\{(w-n)^{2}+2 m\right\}\right\rceil$ is a lower bound if nets are allowed to cross the initial routing window ( $w$ is the width of the window, $n$ is the number of nets and $m$ is the number of nontrivial nets, i.e. those that need to be 'moved'). Following the argument, it is easy to see that $t \geqslant m /(w-n)$ if the nets are not allowed to cross the window. With the concept of channel flux, Baker et al [15] proposed $\max (f, d)$ to be the lower bound. Lee and Leong [16] suggested that for any path $P$ in VCG, if $S_{p}$ is the set of nets not in $P$ but intersect every element in $P$, then $\tau=\max \left(|P|+\left|\operatorname{clique}\left(S_{p}\right)\right|\right)$ is a tighter lower bound for the channel height. We shall re-examine the lower bound with zone representation [17] and VCG for a multiterminal net routing instance. Rigorous details are omitted in the following for the sake of brevity.

IEE Proc.-Comput. Digit. Tech., Vol. 142, No. 4, July 1995
The zone representation does characterise the doglegfree restricted Manhattan routing model for two-terminal nets but is not accurate enough for more general multiterminal net problems with doglegging. Adjacent subnets can either be routed on the same or different track (by adding doglegs), thus occupying one track for the former but two tracks for the latter. This can be represented more accurately by a reduced zone representation (RZR), as illustrated in Fig. $5 b$. The zone representation was originally defined for open interval $\left(z_{1}, z_{2}\right]$ where $z_{1}$ and $z_{2}$ are the positions of two terminal columns acting as the boundary of the zone. In RZR, the interval $\left(z_{1}, z_{2}\right)$ is used instead. Observe that a pair of adjacent subnets which are not merged in the routing solution will have the right subnet appearing in the left zone, e.g. subnet $2 b$ in Fig. $5 b$. Construction of the RZR requires a prior knowledge of whether each pair of adjacent subnets is merged in the final channel routing solution. Fortunately, we can obtain better insight from the VCG. Let us construct a reduced vertical constraint graph (RVCG) by merging all subnet nodes (belonging to the same net) on the VCG into a single node subject to the condition of not elongating the vertical constraint path. Fig. $5 c$ shows the RVCG of the channel routing instance in Fig. $5 a$. Note that subnets $4 a$ and $4 b$ (not shown) are merged, but $2 a$ and $2 b$ are not. If we merge $2 a$ and $2 b$, then $l$ will increase from three to four. Whichever pair of subnets is merged in the RVCG, they will also be merged in the RZR. The RZR density $d_{R}$ is then determined. Finally, we have $t_{R}=\max \left(d_{R}, \tau\right)$ as an improved lower bound. In fact this is the best known lower bound for the channel routing problem. In Fig. $5, d_{R}=4$ and $\tau=3$ thus giving $t_{R}=4$. Fig. $5 a$ shows an optimal solution which requires four tracks to complete the routing.

As we allow doglegging, we consider the channel horizontal contraints with MHCG. To reduce the value of $d_{R}$, we introduce the cost $w_{3}$ in our heuristic cost function described in the following section. The combination of weights $w_{2}$ and $w_{3}$ attempts to lower the value of $t_{R}$ in the final channel routing stage. Next, cycles may occur in a VCG. Without doglegging, a channel with a cyclic VCG does not have a routing solution. In fact cycles in the VCG pose a problem to many channel routers. Fig. $4 b$ shows how OTC routing can provide a means of breaking a cycle. We give preference to candidates that can break any cycle. This is reflected by $w_{1}$ in our cost function.

\subsection{Cost function for OTC routing}

With all the valid OTC routing candidates, we construct a candidate set,

$$
c_{c}=\left\{\left(s_{i, j}, p_{i, j}\right):\left(\exists c_{r_{k}} \in c_{r}\right)\left[l_{i, j} \leqslant c_{r k} \leqslant r_{i, j}\right]\right\}
$$

where $p_{i, j}$ is the OTC routing type for net segment $s_{i, j}$. Two candidates may be conflicting, i.e. routing both over the cells will cause a short circuit. For example, type-1 of Net 2 and type- 2 of Net 3 in Fig. $1 c$ are conflicting. We use $f_{c}\left(s_{i, j}, p_{i, j}\right)$ to denote the number of candidates conflicting with the candidate $\left(s_{i, j}, p_{i, j}\right)$. Minimal conflicts allow more net segments to be routed OTC. Now a proper candidate in $c_{c}$ has to be selected for routing OTC in every iteration. To make this selection, we device four weights below to calculate the criticality of each candidate.

$$
\begin{aligned}
w_{1} & =1 \quad \text { if routing }\left(s_{i, j}, p_{i, j}\right) \text { OTC will break a cycle } \\
& =0 \text { otherwise }
\end{aligned}
$$



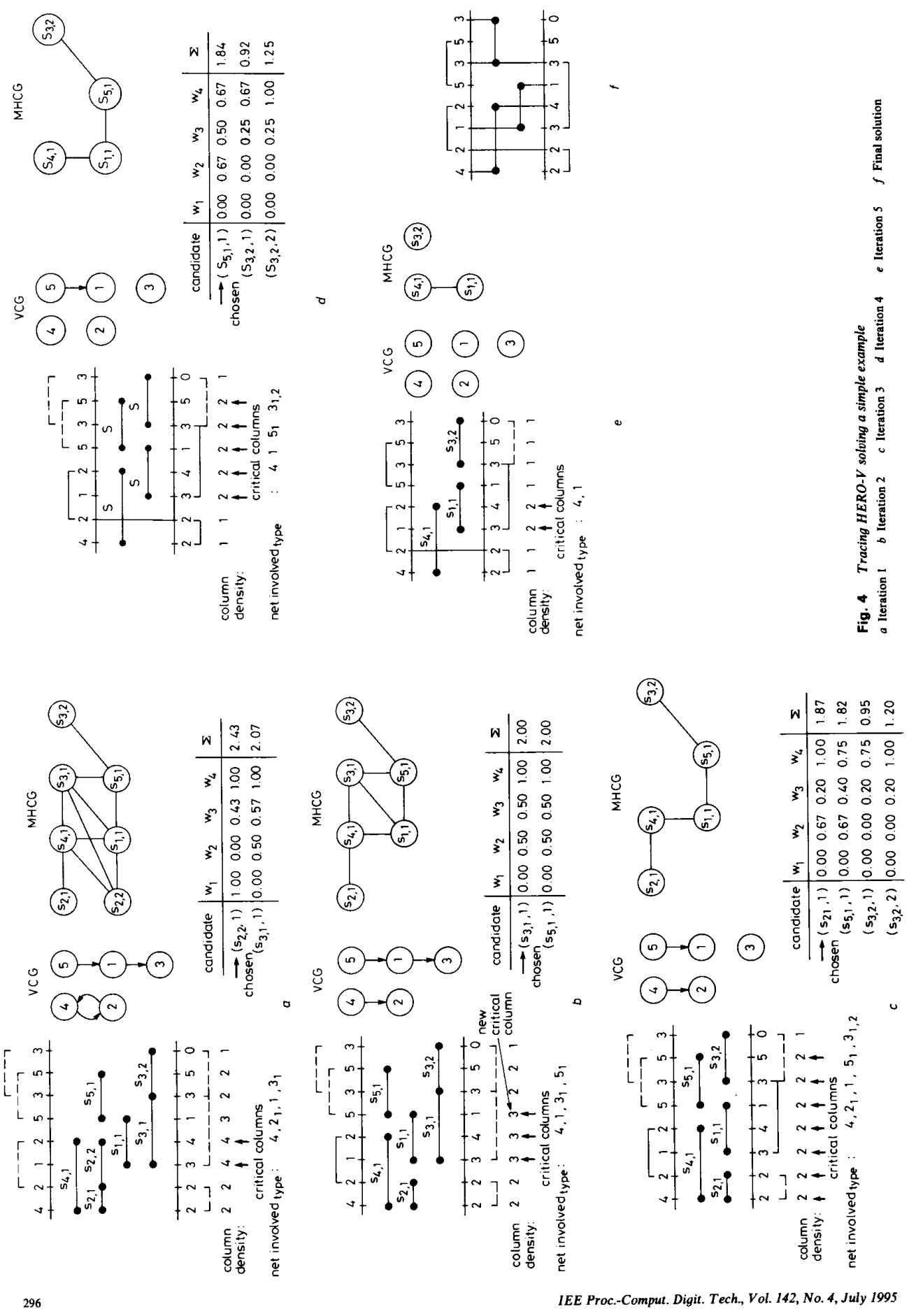


$$
\begin{aligned}
w_{2} & =0 \quad \text { if } n_{i} \notin L_{V G} \\
& =1-\frac{\left|2 l_{r}-l_{0}-1\right|}{l_{0}+1} \text { otherwise } \\
w_{3} & =\frac{d\left(v_{i, j}, G_{m k}\right)}{\left|V^{\prime}\right|} \\
w_{4} & =1-\frac{f_{c}\left(s_{i, j}, p_{i, j}\right)}{\left|c_{c}\right|}
\end{aligned}
$$

Here $L_{V G}$ is the longest path in the vertical constraint graph and $l_{0}$ is the length of the longest path, while $l_{r}$ is the relative position of the candidate net in the longest path, $d(v, G)$ is the degree of vertex $v$ in the undirected graph $G$.

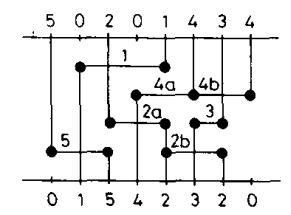

Fig. 5A Channel routing instance

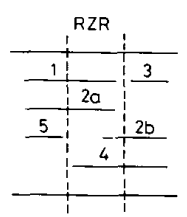

Fig. 5B Reduced zone representation

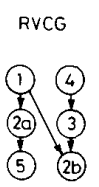

Fig. 5C Reduced vertical constraint graph

\section{Summary of our algorithm}

The essence of our algorithm lies in the selection of net segments to be routed over the cells. The algorithm can be summarised in the following steps:

(a) Decompose multiterminal nets: All multiterminal nets are decomposed into two-terminal nets adjacent at common terminal locations thus giving us a set of twoterminal net segments.

(b) Determine critical columns and OTC candidates: The channel density is determined before the set of critical columns $c_{r}$ is constructed. From the critical columns and based on the valid OTC routing types (see Section 4.1), we construct a set of OTC candidates which are not conflicting with those already routed OTC net segments. Conflicting ones are discarded and will not be considered further. When the corresponding net segment of the selec-

IEE Proc.-Comput. Digit. Tech., Vol. 142, No. 4, July 1995 ted candidate is removed from the channel in step (d), the column densities within its span are decreased by one and more columns become critical. Hence $c_{r}$ and, similarly, the set of OTC candidates are updated in subsequent iterations.

(c) Determine $L_{V G}$ and find cycle-breaking nodes in $V G$ : We construct the vertical constraint graph $V G$ and determine the set of longest path(s) $L_{V G}$. If cycles occur, we find the valid candidates that can break a cycle in $V G$, if they are routed OTC.

(d) Weight assignment and OTC candidate selection: The criticality of each OTC candidate is calculated by summing the four weights described in Section 4.1. The most critical net segment will be selected. In the case of equal weights, we choose the left-most candidate.

(e) Track assign selected OTC candidate: The selected candidate is then track-assigned in the OTC area. We do not perform the actual routing in this step because assigning a new selected net segment may involve shifting previously assigned segments. The final geometrical routing will be done in step $(g)$. Next, the corresponding net segment of the selected candidate is removed from the channel.

(f) Repeat step (b) until no more net segment can be routed over the cells: Once a critical net segment is removed from the channel in step (e), we update the column (channel) densities. The VCG, as well as the MHCG will also be updated.

(g) Route track assigned OTC net segments: Assignment of geometrical track location for each OTC net segment is finalised after the above iteration. The OTC net segments are routed accordingly here. This step marks the end of the OTC routing phase.

(h) Route remaining net segments in channel: Net segments not routed OTC will be routed in this step using conventional channel routing techniques.

The differences between two-layer and three-layer OTC routing occurs mainly in steps $(b)$ to $(d)$. For each layer of metal available a disjoint candidate is selected, hence in three-layer OTC routing an M2 candidate and an M3 candidate will be selected. The enumeration of an OTC candidate set and its maintenance in step $(b)$ contributes the highest computational complexity. The overall OTC routing is of the order of $O\left(n^{2} k^{2} l\right)$ where $n$ is the number of net, $k$ is the maximum number of net interval of a net and $l$ is the maximum length (in terms of columns) of a net interval.

After the OTC routing phase, the remaining net segments are routed in the channel by a conventional channel router. We use an HV model for two-layer routing and an $\mathrm{HVH}$ model for three-layer routing in the channel. The routing problem left in the channel is much simplified as most cycles and vertical constraints are removed in the OTC routing phase.

\section{Experimental results}

We have implemented our algorithm in the $C$ language on a Sun SPARC 10 station running SunOS 4.1.3. We call our program HERO-V. It was tested extensively on common benchmark examples for channel routing problem including the Deutsch's Difficult Example. Table 1 shows the results for the PRIMARY 1 benchmark compared to other algorithms, including the Greedy router [1] (denoted Gr in Table 1), OTC-I [10], WISER [8,9], WILMA [12] and O-V [11]. Results shown for a threelayer model assume vias are not permitted on the area 
over the cells. The best result for each channel is marked with an asterisk. Table 2 shows the results compared to those in References 3 and 5. HERO-V is comparable to

Table 2: Comparison with other routers in References 3 and 5 for a two-layer model

\begin{tabular}{|c|c|c|c|c|c|c|}
\hline \multirow[t]{2}{*}{ Example } & \multirow{2}{*}{$\begin{array}{l}\text { Channel } \\
\text { length }\end{array}$} & \multirow{2}{*}{$\begin{array}{l}\% \text { vacant } \\
\text { terminal }\end{array}$} & \multirow{2}{*}{$\begin{array}{l}\text { Original } \\
\text { density }\end{array}$} & \multicolumn{3}{|c|}{ Final channel height } \\
\hline & & & & [3] & [5] & HERO-V \\
\hline Ex1 & 43 & 24 & 12 & 10 & 9 & 9 \\
\hline Ex3a & 89 & 33 & 15 & 15 & 12 & 11 \\
\hline Ex3b & 84 & 22 & 17 & 16 & 13 & 14 \\
\hline Ex $\times$ c & 103 & 26 & 18 & 16 & 15 & 15 \\
\hline Ex4b & 119 & 24 & 17 & 16 & 12 & 13 \\
\hline & 128 & 35 & 20 & 14 & 12 & 11 \\
\hline Deutsch & 175 & 14 & 19 & 20 & 17 & 17 \\
\hline Total & - & 25 & 118 & 107 & 90 & 90 \\
\hline
\end{tabular}

[5] on these benchmark examples. To check the performance on circuits with less vacant terminals, we tested the algorithm with three channels from a register decoder circuit. The results are shown in Table 3 . The time taken for each execution is also indicated. With regard to the execution time, the average time taken in OTC routing one of the PRIMARY 1 channel was $19.15 \mathrm{~s}$. Each of the PRIMARY 1 and our decoder benchmark examples has the channel length of 477 and 118 , respectively.

Table 3: Results for decoder benchmarks and the execution times

\begin{tabular}{|c|c|c|c|c|c|c|c|}
\hline \multirow[t]{2}{*}{ Channel } & \multirow[t]{2}{*}{ Vacant } & \multirow{2}{*}{$\begin{array}{l}\% \\
\text { Vacancy }\end{array}$} & \multirow{2}{*}{$\begin{array}{l}\text { Original } \\
\text { density }\end{array}$} & \multicolumn{2}{|c|}{ Two-layer } & \multicolumn{2}{|c|}{ Three-layer } \\
\hline & & & & $\begin{array}{l}\text { Final } \\
\text { channel } \\
\text { height }\end{array}$ & $\begin{array}{l}\text { Time } \\
\text { (s) }\end{array}$ & $\begin{array}{l}\text { Final } \\
\text { channel } \\
\text { height }\end{array}$ & $\begin{array}{l}\text { Time } \\
\text { (s) }\end{array}$ \\
\hline $\begin{array}{l}\mathrm{D} 1 \\
\mathrm{D} 2\end{array}$ & $\begin{array}{r}104 \\
83\end{array}$ & $\begin{array}{l}44 \\
35\end{array}$ & $\begin{array}{l}16 \\
16\end{array}$ & $\begin{array}{l}12 \\
13\end{array}$ & $\begin{array}{l}2.23 \\
0.46\end{array}$ & $\begin{array}{l}5 \\
5\end{array}$ & $\begin{array}{l}3.86 \\
1.12\end{array}$ \\
\hline D3 & 103 & 44 & 15 & 10 & 1.23 & 4 & 3.81 \\
\hline
\end{tabular}

Amongst the routers, the Greedy channel router does not use OTC are while the rest do. Comparison in Table 1 also illustrates the significance of routing over the cells. In terms of total channel height, HERO-V achieved a $41.3 \%$ improvement over the Greedy router for the twoplayer model and a $61.0 \%$ improvement for the threelayer model. To emphasise this, we notice the total channel height obtained by HERO-V for two-layer routing (175 tracks) is approaching that obtained by Greedy channel router using three-layer routing (172 tracks). Note that the channels in PRIMARY 1 , as in many practical layouts, have many vacant terminals (on the average $65 \%$ of the terminals are vacant). From our experience, the improvement in channel height is most promising when a channel has many vacant terminals as our approach can make use of them efficiently to reduce the channel height.

\section{Conclusions}

In this paper we have presented a new algorithm for the over-the-cell channel routing problem in standard cell
VLSI design. Our approach can efficiently reduce the channel height especially when there are many vacant terminals in the channel. It has achieved the best results in the PRIMARY 1 benchmark examples compared to previous algorithms.

Our algorithm is suitable for routing problems in which vias are not permitted on the area over the cells. We are currently developing algorithms for the case when vias are permitted. We hope the new algorithms will render better results in the future.

\section{References}

1 RIVEST, R.L., and FIDUCCIA, C.M.: 'A "greedy" channel router'. Proceedings of 19th ACM/IEEE Conference on Design Automation 1982, pp. 418-424

2 SZYMANSKI, T.G.: 'Dogleg channel routing is NP complete', IEEE Trans., 1985, CAD-4, pp. 31-41

3 SHIRAISHI, Y., and SAKEMI, Y.: 'A permeation router', IEEE Trans., 1987, CAD-6, pp. 462-471

4 CONG, J., and LIU, C.L.: 'Over-the-cell channel routing', IEEE Trans., 1990, CAD-9, (4), pp. 408-418

5 CONG, J., PREAS, B., and LIU, C.L.: 'Physical models and efficient algorithms for over-the-cell routing in standard cell design', IEEE Trans., 1993, CAD-12, (5), pp. 723-734

6 PAI, R.R., and RAO, S.S.S.P.: 'An over-the-cell channel router' Proceedings of IFIP TC10/WG 10.5 International Conference on $V L S I, 1991$, pp. $327-336$

7 LIN, M.S., PERNG, H.W., HWANG, C.Y., and LIN, Y.L. 'Channel density reduction by routing over the cells'. Proceedings of 28th ACM/IEEE Conference on Design automation, 1991, pp. 120125

8 HOLMES, N.D., SHERWANI, N.A., and SARRAFZADEH, M.: New algorithm for over-the-cell channel routing using vacant terminals'. Proceedings of 28th ACM/IEEE Conference on Design automation, 1991, pp. 126-131

9 HOLMES, N.D., SHERWANI, N.A., and SARRAFZADEH, M.: 'Utilization of vacant terminals for improved over-the-cell channel routing', IEEE Trans., 1993, CAD-12, (6), pp. 780-792

10 CHANG, L.D., HSIAO, P.Y., YAN, J.T., and SHEW, P.W.: 'A robust over-the-cell channel router', IEEE Trans., 1993, CAD-12, (10), pp. 1592-1599

11 HOLMES, N.D., SHERWANI, N.A., and SARRAFZADEH, M.: 'Algorithms for three-layer over-the-cell channel routing'. Proceedings of International Conference on Computer-aided Design, 1991, pp. 428-431

12 NATARAJAN, S., SHERWANI, N., HOLMES, N.D., and SAR. RAFZADEH, M.: "Over-the-cell channel routing for high performance circuits' Proceedings of 29th ACM/IEEE Conference on Design Automation, 1992, pp. 600-603

13 DEUTSCH, D.N.: 'A dogleg channel router'. Proceedings of 13th ACM/IEEE Conference on Design Automation, 1976, pp. 425-433

4 BROWN, D J, and RIVEST, R L. "New lower bounds for channel width' Proceedings of CMU Conference on VLSI systems and comwidth'. Proceedings of CM

15 BAKER, B.S., BHATT, S.N., and LEIGHTON, F. T. 'An approximation algorithm for Manhattan routing'. 15th Annual Symposium on Theory of computing 1983

16 LEE, K.K and LEONG, H.W. 'An improved lower bound for channel routing problems'. Proceedings of International Symposium on Circuits and systems, 1991, pp. 1952-1955

17 YOSHIMURA, T., and KUH, E.S.: 'Efficient algorithms for channel routing', IEEE Trans., 1982, CAD1, pp. 25-35 